CASE REPORT

\title{
Elusive tuberculous meningitis with rare neurological complication of longitudinally extensive transverse myelitis: a case report
}

\author{
Zaitoon Zafar ${ }^{1}$, Muhammad Hassan Hafeez $\mathbb{D}^{1 凶}$ and Mujeeb Ur Rehman Abid Butt ${ }^{2}$ \\ (c) The Author(s), under exclusive licence to International Spinal Cord Society 2021
}

\begin{abstract}
INTRODUCTION: Longitudinally extensive transverse myelitis (LETM) is inflammation of the spinal cord that spans three or more spinal segments. LETM is a rare occurrence on its own and has seldom been reported with tuberculous meningitis (TBM), the rarest and deadliest of tuberculous manifestations. TBM is usually seen in children, the immunocompromised, or those with a previous history of tuberculosis infection.

CASE PRESENTATION: A 24-year-old healthy male with no co-morbidities or history of tuberculosis presented with fever and headache for the past 3 months. The patient's Kernig's and Brudzinski's signs were both negative, with bilateral abnormal plantar reflexes. The neurological level of injury was T8 and the patient was classified as AIS grade A. His CSF analysis showed a lymphocytic picture. However, both GeneXpert and Ziehl-Neelsen staining came back negative for Mycobacterium tuberculosis. MRI scans of the brain and thoracic spine revealed enhancing nodules and ring lesions in the brain and spinal cord, along with the rare complication of LETM, extending from T2 to T9.

DISCUSSION: Although Mycobacterium tuberculosis was never isolated, the patient started recovering as soon as antituberculous therapy was initiated. Hence, more emphasis needs to be placed on radiological imaging in the management of rare medical emergencies like tuberculous meningitis, especially in areas where tuberculosis is rampant and endemic, rather than waiting for a positive culture. This case report also demonstrates the growing evidence that transverse myelitis and/or LETM is associated with TBM.
\end{abstract}

Spinal Cord Series and Cases (2021)7:82; https://doi.org/10.1038/s41394-021-00445-y

\section{INTRODUCTION}

The World Health Organization's Global Tuberculosis Report 2020 lists Tuberculosis (TB) as one of the top ten causes of death worldwide and the leading cause of death from a single infectious agent, ranking even higher than HIV [1]. Tuberculous meningitis (TBM) is a form of extrapulmonary TB characterized by inflammation of the meninges of the brain and spinal cord due to the bacillus Mycobacterium TB and is considered a medical emergency [2]. TBM is a rare disorder, encompassing a total of $1 \%$ of tuberculous manifestations [3] and is also considered the deadliest of all tuberculous presentations, imparting lifelong morbidity and significant mortality $[4,5]$. The rare complication of transverse myelitis affects $\sim 5$ per million people every year, and its association with TBM goes largely unreported [6]. Here, we present the case of a young adult male who presented to a tertiary care hospital in Lahore, Pakistan, with a challenging and elusive diagnosis of TBM, in addition to the rare complication of longitudinally extensive transverse myelitis (LETM).

\section{CASE PRESENTATION}

A 24-year-old male presented to the Emergency Room with fever and headache for the past 3 months. The patient only reported to the hospital when he developed neck stiffness, an altered state of consciousness, muscle spasms, and a solitary seizure. His fever gradually reached $103^{\circ} \mathrm{F} 4$ days before presentation, associated with an increase in the severity of his headache, and nuchal rigidity. The patient claimed his headache was aggravated by light, and also complained of constipation, in addition to difficulty with urination.

The patient appeared irritable, and a Glasgow Coma Scale of 12/ 15 was recorded at the time of admission. An emergency chest $x$-ray revealed no abnormal findings and no signs of consolidation. Upon examination, the patient's Kernig's and Brudzinski's signs were both negative. He had flaccid paralysis in his lower extremities, and bilateral abnormal plantar reflexes (Babinski reflex).

A sensory and motor examination was performed and, in accordance with the ASIA/ISCOS International Standards for Neurological Classification of Spinal Cord Injury (ISNCSCI), his sensory level was determined to be T8 with a score of $1 / 1$ on both sides and total sensory sub-scores were 58/112, for both light touch and pin-prick sensations. A Muscle Function Grading score of $0 / 5$ in both his lower limbs was noted, with a total Lower Extremity Motor sub-score of $0 / 50$. The Neurological Level of Injury

\footnotetext{
'Department of Medicine, Shalamar Medical and Dental College, Lahore, Pakistan. ${ }^{2}$ Department of Medicine, Shalamar Hospital, Lahore, Pakistan. email: hassanhafeez911@gmail.com
}

Received: 12 February 2021 Revised: 6 September 2021 Accepted: 6 September 2021

Published online: 14 September 2021 

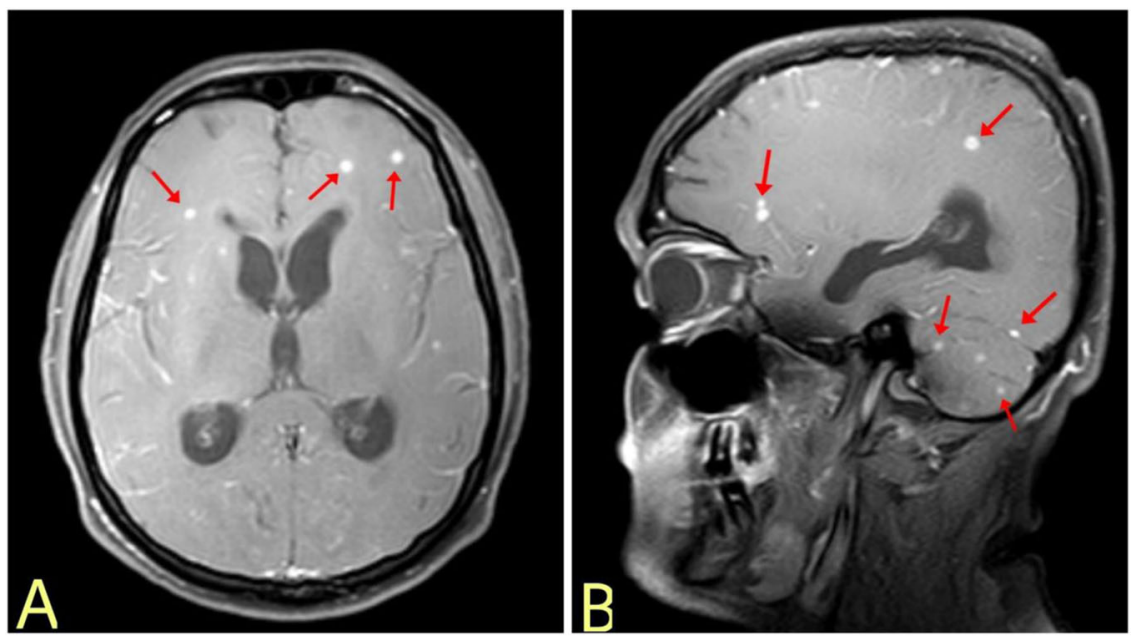

Fig. 1 Brain MRI. T1 weighted MRI scans of the brain with contrast in axial (A) and sagittal (B) planes reveal basal meningeal enhancement, red arrows point to ring lesions at varying positions in the brain.

was 78 , with the absence of voluntary anal contraction and deep anal pressure. This classified the patient as AIS (ASIA Impairment Scale) grade $A$, indicating complete spinal cord injury (Supplementary ASIA Worksheet).

The patient was admitted to the Intensive Care Unit (ICU) and kept under strict monitoring. His PCR was negative for SARS-CoV2. An electrolyte panel revealed hyponatremia of $125 \mathrm{mEq} / \mathrm{L}$. Labs showed a C-reactive protein level of $84.2 \mathrm{mg} / \mathrm{L}$ and blood and sputum culture identified no microorganisms. A GeneXpert test was conducted, which also came back negative for Mycobacterium TB. A CT scan of the brain revealed hydrocephalus and dilation of the ventricles; high intracranial pressure contraindicated a lumbar puncture. Papilledema was noted on ophthalmological examination. The patient was given Acyclovir, as HSV was suspected, with no improvement to his condition.

Magnetic Resonance Imaging (MRI) of the brain and thoracic spine with gadolinium contrast was conducted, along with a highrisk lumbar puncture; with informed consent from the patient. Brain MRI scans (Fig. 1) noted enhancing nodules and ring lesions in both the cerebral hemispheres and the cerebellum along with basal meningeal enhancement. MRI scan of the spinal cord (Fig. 2) revealed ring-enhancing lesions in the intramedullary compartment of the upper dorsal spinal cord with extensive surrounding edema, along with LETM extending from thoracic segment T2 to T9.

Cerebrospinal fluid (CSF) was yellow-colored with a $99 \%$ lymphocytic picture, a high protein level of $298 \mathrm{mg} / \mathrm{dL}$, and a low sugar level of $21 \mathrm{mg} / \mathrm{dL}$. However, Gram and Ziehl-Neelsen staining did not detect any organism or acid-fast bacilli, respectively. His CSF was also negative for monoclonal bands and aquaporin-4 antibodies. The patient's tests for HSV, HIV, syphilis, and autoimmune markers also came back negative.

Despite no organism being detected, TBM was suspected, based on CSF findings, and the patient was promptly treated with four daily doses of Myrin P-Forte (a combination of Ethambutol HCL 275 mg, Rifampicin $150 \mathrm{mg}$, Isoniazid $75 \mathrm{mg}$, and Pyrazinamide $400 \mathrm{mg}$ ) and Vita-6 (a vitamin B6 preparation) $50 \mathrm{mg}$ oral tablets, in addition to Dexamethasone $10 \mathrm{mg}$ stat, followed by $4 \mathrm{mg} 6 \mathrm{~h}$.

The patient's sensorium improved considerably within a day of treatment and he was soon shifted from the ICU to the High Dependency Unit. A Mini-Mental State Examination was performed to assess the patient's cognitive functioning, on which he scored 30/30. He still had weakness in his lower extremities and bilateral abnormal plantar reflexes, for which physiotherapy was advised, and the patient was discharged soon after. The patient was bed bound and catheterized at the time of discharge.
Keeping in line with the guidelines in place for tuberculous infections, the patient's family members were advised a Mantoux test and a chest X-ray, none of them tested positive and/or showed any signs of a tuberculous infection.

On monthly follow-up in the out-patient clinic, the patient appeared to be fully alert with mildly increased tone in his lower limbs. He was found to be mobile with forearm crutches and with minimal support from his attendants. Knee and ankle reflexes were mildly brisk with a positive Babinski sign on both plantars. The patient's sensory level was still at T8 bilaterally. However, the patient's AIS grade improved to D (according to the ISNCSCI) as his Muscle Function Grade had increased to $3 / 5$ (previously $0 / 5$ ) in the lower limbs (Supplementary ASIA Worksheet).

At 3 months follow-up, the patient had Muscle Function Grading of $3+/ 5$, he was ambulating with forearm crutches, and required minimal support from his family for his routinely tasks such as changing his clothes. The patient continued with a catheter, but reported to have some self-control on his bowel activity and his constipation also improved to a reasonable level. The patient was compliant with the prescribed course of antituberculous therapy and physiotherapy sessions, his AIS score and sensory levels were consistent with the previous monthly findings.

\section{DISCUSSION}

The clinical presentation of TBM is similar to other causes of meningitis with fever, headache, neck rigidity, photophobia, vomiting, altered sensorium, and positive clinical findings upon examination, with the diagnosis being confirmed upon CSF culture $[3,7]$. Our patient's presentation was curious, in that both Kernig's and Brudzinski's signs were negative and Mycobacterium $T B$ was never isolated. Furthermore, TBM occurs more commonly in children, in the immunocompromised, or those with a previous history of tuberculous infection [3, 4]. Our patient, however, is a healthy young immunocompetent adult with no co-morbidities, not living in a crowded space. The only remarkable finding in his medical history was a Hepatitis A infection over a year ago, which resolved without any complications.

TBM is associated with a number of intracranial complications including cranial nerve palsies, hydrocephalus, vasculitic infarction, tuberculoma formation, and hyponatremia; as well as spinal complications including spinal tuberculoma, syringomyelia, and acute transverse myelitis (ATM) [7, 8]. Intramedullary lesions, as 

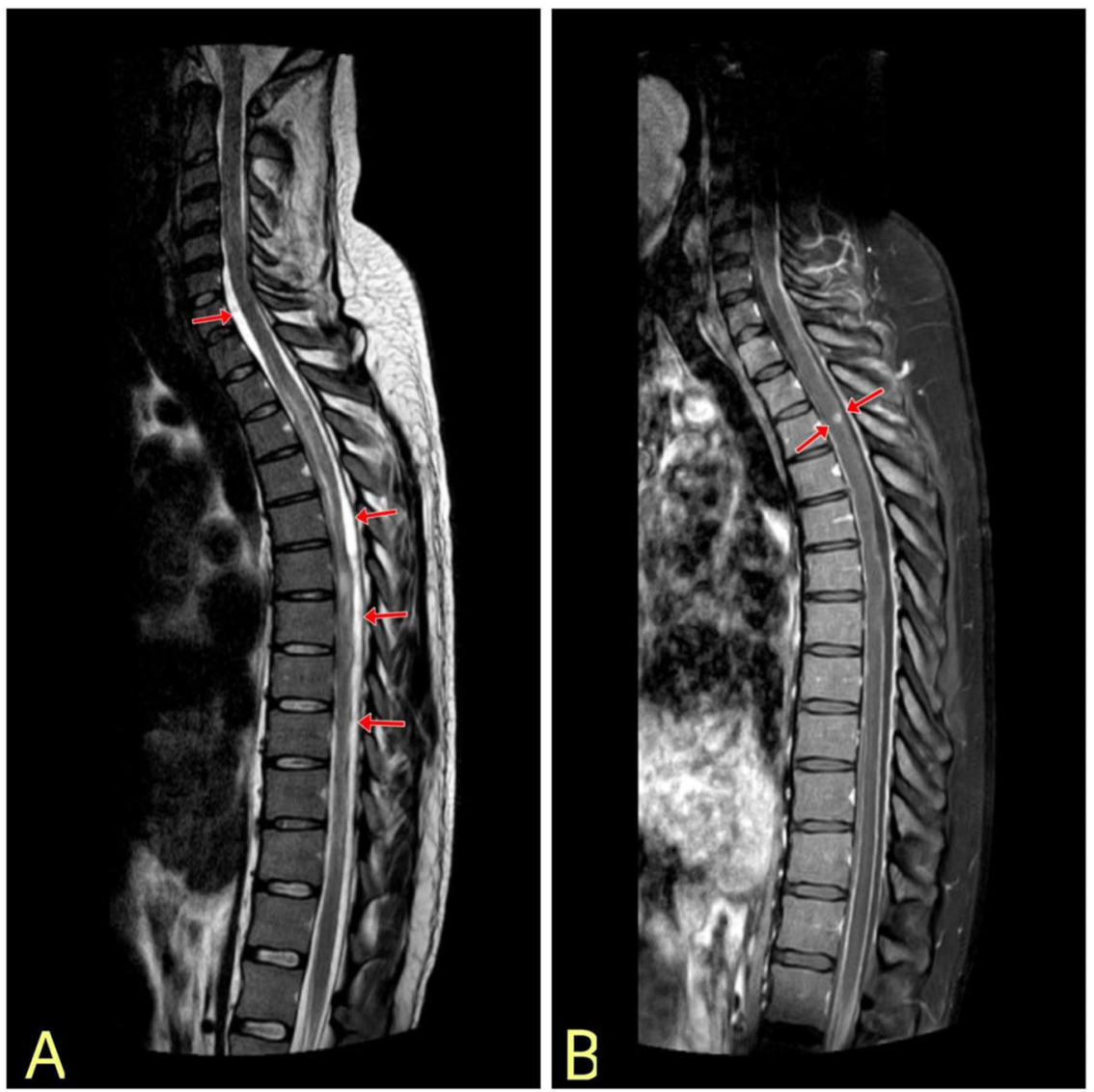

Fig. 2 Spinal Cord MRI. A Sagittal T2-weighted contrast MRI of the spinal cord shows intense edema extending from level T2 to T9, classifying the case as LETM, (B) Sagittal contrast MRI scan shows ring-enhancing lesion in the intramedullary compartment of the upper thoracic spinal cord.

seen in our patient (Fig. 2), manifest in only $7 \%$ of patients with central nervous system TB [9].

Other intracranial manifestations in our patient included hydrocephalus as evident on CT and MRI scans, and daily electrolyte panels confirming hyponatremia. MRI with contrast of the brain showed multiple nodules and ring-enhancing lesions in the brain (Fig. 1). There was no evidence of vascular infarct, hemorrhage, or brainstem abnormality.

Acute transverse myelitis (ATM) is identified as inflammation of the spinal cord with the involvement of one or two spinal segments and it occurs in 1.2-4.6 per million people annually [6]. Longitudinally extensive transverse myelitis (LETM) is distinguished from ATM as it spans three or more spinal segments [8]. The MRI scan of the spinal cord (Fig. 2) reveals intramedullary ring lesions and significant edema, in addition to longitudinally extensive hyperintense lesions extending from T2 to T9 evident on T2-weighted image, consistent with LETM.

Transverse myelitis is usually associated with viral agents, autoimmune disorders, or adverse drug reactions. An immunologic reaction is thought to be explicitly accountable for the demyelinating inflammation seen in LETM $[6,10]$.

TBM has rarely been associated with transverse myelitis [6$8,10,11]$, and a previous case report owes the exaggerated presentation of LETM in association with TBM to a paradoxical response [11]. According to a hypothesis, the release of the tubercular protein upon administration of ATT triggers an intense inflammatory response and worsens pre-existing inflammation of the spine. Although the paradoxical response has a propensity for the central nervous system [12], we would like to emphasize that our patient presented with untreated LETM with no prior history of TB infection or any co-morbidity, and showed improvement upon ATT administration.

The patient's CSF culture and Genexpert tests came back negative for Mycobacterium TB. Viral, autoimmune and syphilitic causes were ruled out during investigations. Demyelinating disorders like multiple sclerosis and neuromyelitis optica, the most common differentials of LETM [13], were also ruled out by testing CSF for monoclonal bands and aquaporin-4 antibodies, respectively.

Radiological and laboratory findings of nodules, ring enhanced lesions, LETM, and hyponatremia in addition to a classical CSF picture of TBM [14] with lymphocytic pleocytosis, raised protein levels, and low glucose levels heightened our suspicion of TBM, and the patient's prompt response to ATT confirmed our suspicion. The diagnosis is in line with the guidelines proposed by Lee et al. [15], and with the "Thwaites' scoring system" [16], but not in accordance with the scoring system proposed in The Lancet [17].

\section{CONCLUSIONS}

Owing to TBM being a medical emergency, we recommend not waiting for a positive Mycobacterium TB culture and starting antituberculous therapy as soon as suspicion for TBM is raised, with $C T$ and MRI scans being first-line investigations. The scoring system put forward by Lee et al. to differentiate between TBM and viral meningitis is also recommended and will be used in our clinical setting from now on, as Pakistan is one of the eight countries responsible for two-thirds of global TB cases. Our patient's recovery has been appreciable despite the condition left untreated and worsening for more than 3 months. We owe the 
patient's lack of permanent morbidity to quick treatment upon seeking medical assistance, in addition to the patient's compliance with the treatment regimen, which we have prescribed for 6 months continuously, subject to review based on the patient's recovery.

\section{REFERENCES}

1. World Health Organization: Global tuberculosis report 2020. https://www.who. int/publications/i/item/9789240013131. Accessed: December 11, 2020.

2. Thwaites G, Fisher M, Hemingway C, Scott G, Solomon T, Innes J. British Infection Society guidelines for the diagnosis and treatment of tuberculosis of the central nervous system in adults and children. J Infect. 2009;59:167-87. https://doi.org/ 10.1016/j.jinf.2009.06.011.

3. Slane VH, Unakal CG. Tuberculous Meningitis. In: StatPearls [Internet]. Treasure Island (FL): StatPearls Publishing; 2020 Jan-. https://www.ncbi.nlm.nih.gov/books/ NBK541015/.

4. Van TT, Farrar J. Tuberculous meningitis. J Epidemiol Community Health. 2013;68:195-6. https://doi.org/10.1136/jech-2013-202525.

5. Soria J, Metcalf T, Mori N, Newby RE, Montano SM, Huaroto L, et al. Mortality in hospitalized patients with tuberculous meningitis. BMC Infect Dis. 2019;19:9 https://doi.org/10.1186/s12879-018-3633-4.

6. Bhat A, Naguwa S, Cheema G, Gershwin ME. The epidemiology of transverse myelitis. Autoimmun Rev. 2010;9:A395-9. https://doi.org/10.1016/j. autrev.2009.12.007.

7. Schaller MA, Wicke F, Foerch C, Weidauer S. Central Nervous System Tuberculosis: Etiology, Clinical Manifestations and Neuroradiological Features. Clin Neuroradiol. 2018;29:3-18. https://doi.org/10.1007/s00062-018-0726-9.

8. Garg RK, Malhotra HS, Gupta R. Spinal cord involvement in tuberculous meningitis. Spinal Cord. 2015;53:649-57. https://doi.org/10.1038/sc.2015.58.

9. Nussbaum ES, Rockswold GL, Bergman TA, Erickson DL, Seljeskog EL. Spinal tuberculosis: a diagnostic and management challenge. J Neurosurg. 1995;83:243-7. https://doi.org/10.3171/jns.1995.83.2.0243.

10. Kitley JL, Leite MI, George JS, Palace JA. The differential diagnosis of longitudinally extensive transverse myelitis. Mult Scler. 2012;18:271-85. https://doi. org/10.1177/1352458511406165.

11. Jain RS, Kumar S, Tejwani S. A rare association of tuberculous longitudinally extensive transverse myelitis (LETM) with brain tuberculoma. Springerplus. 2015;4:476 https://doi.org/10.1186/s40064-015-1232-z.

12. Cheng VC, Ho PL, Lee RA, Chan KS, Chan KK, Woo PC, et al. Clinical spectrum of paradoxical deterioration during antituberculosis therapy in non-HIV-infected patients. Eur J Clin Microbiol Infect Dis. 2002;21:803-9. https://doi.org/10.1007/ s10096-002-0821-2.
13. Trebst C, Raab P, Voss EV, Rommer P, Abu-Mugheisib M, Zettl UK, et al. Longitudinal extensive transverse myelitis-it's not all neuromyelitis optica. Nat Rev Neurol. 2011;7:688-98. https://doi.org/10.1038/nrneurol.2011.176.

14. Chaidir L, Ganiem AR, Vander Zanden A, Muhsinin S, Kusumaningrum T, Kusumadewi I, et al. Comparison of Real Time IS6110-PCR, Microscopy, and Culture for Diagnosis of Tuberculous Meningitis in a Cohort of Adult Patients in Indonesia. PLoS ONE. 2012;7:52001 https://doi.org/10.1371/journal.pone.0052001.

15. Lee SA, Kim SW, Chang HH, Jung H, Kim Y, Hwang S, et al. A New Scoring System for the Differential Diagnosis between Tuberculous Meningitis and Viral Meningitis. J Korean Med Sci. 2018;33. https://doi.org/10.3346/jkms.2018.33.e287.

16. Thwaites GE, Chau TT, Stepniewska K, Phu NH, Chuong LV, Sinh DX, et al. Diagnosis of adult tuberculous meningitis by use of clinical and laboratory features. Lancet 2002;360:1287-92. https://doi.org/10.1016/S0140-6736(02) 11318-3.

17. Marais S, Thwaites G, Schoeman JF, Török ME, Misra UK, Prasad K, et al. Tuberculous meningitis: a uniform case definition for use in clinical research. Lancet Infect Dis 2010;10:803-12. https://doi.org/10.1016/S1473-3099(10)70138-9.

\section{AUTHOR CONTRIBUTIONS}

All authors contributed equally. ZZ, MHH, MRAB gathered and interpreted the data, performed the literature search and wrote the paper. All authors revised and approved the final submission.

\section{COMPETING INTERESTS}

The authors declare no competing interests.

\section{ADDITIONAL INFORMATION}

Supplementary information The online version contains supplementary material available at https://doi.org/10.1038/s41394-021-00445-y.

Correspondence and requests for materials should be addressed to Muhammad Hassan Hafeez.

Reprints and permission information is available at http://www.nature.com/ reprints

Publisher's note Springer Nature remains neutral with regard to jurisdictional claims in published maps and institutional affiliations. 